\title{
Hemichorea-Hemiballism as the First Presentation of Type 2 Diabetes
}

Sayantan Ray, ${ }_{1}$ Sukharanjan Howlader, ${ }^{2}$ Sumit Chakraborty, ${ }^{2}$ Partha Pratim Chakraborty, and Sujoy Ghosh'1

${ }^{1}$ Department of Endocrinology and

${ }^{2}$ Department of Radiology, Institute of Post Graduate Medical Education \& Research and SSKM Hospital, Kolkata, West Bengal, India

Corresponding author: Sayantan Ray, sayantan.ray30@gmail.com

DOI: 10.2337/diaclin.33.2.87

(C)2015 by the American Diabetes Association. Readers may use this article as long as the work is properly cited, the use is educational and not for profit, and the work is not altered. See http:/l creativecommons.org/licenses/by-nc-nd/3.0 for details.

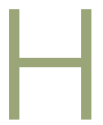
e michorea-he miballis m $(\mathrm{HCHB})$ is a hyperkinetic disorder characterized by continuous, nonpatterned, proximal, involuntary movements on one side of the body, resulting from involvement of the contralateral basal ganglia and particularly the striatum. We report here an interesting case of sudden onset $\mathrm{HCHB}$ associated with nonketotic hyperglycemia, with complete remission of symptoms after control of blood glucose. HCHB as the first presentation of type 2 diabetes has been described rarely. This case report reinforces that, especially in elderly patients with newly diagnosed $\mathrm{HCHB}$, nonketotic hyperglycemia should be recognized promptly. Early diagnosis and treatment of hyperglycemia yield an excellent prognosis.

\section{Presentation}

A 61-year-old man presented after 2 days' duration of sudden-onset involuntary movements of the left side, first involving the upper limb and 6 hours later involving the homolateral lower limb. There was no history of stroke or preceding trauma. He was conscious and alert, with normal higher mental functions and cranial nerve examination. He had periodic choreiform and ballistic movements of the left upper and lower extremities that could not be suppressed voluntarily but that ceased during sleep.

Initial biochemistry revealed a random blood glucose of $527 \mathrm{mg} / \mathrm{dL}$, a normal venous $\mathrm{pH}$ (7.37), and negative blood ketones $(0.5 \mathrm{mmol} / \mathrm{L})$.
A diagnosis was made of diabetic nonketotic hyperosmolar state causing $\mathrm{HCHB}$. The patient's $\mathrm{A} 1 \mathrm{C}$ was $10.3 \%$, consistent with a prolonged
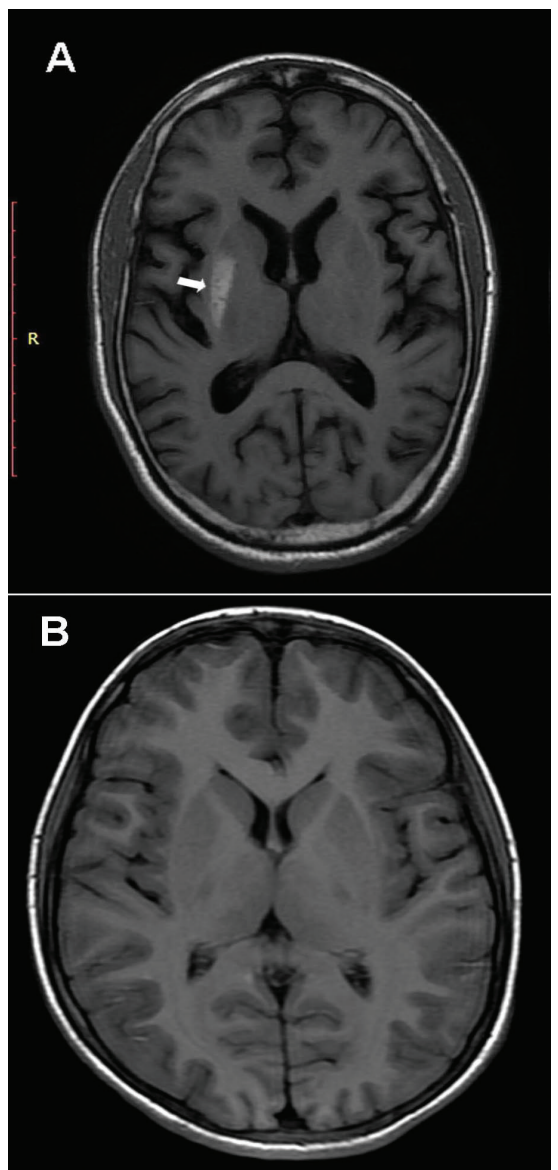

FIGURE 1. (A) An MRI T1-weighted axial image showing an irregular area of high signal intensity involving the posterior right putamen without any appreciable adjacent edema or mass effect. (B) A 6-month re-scan shows complete disappearance of the putaminal lesion. 
period of undiagnosed type 2 diabetes. An MRI study of the brain was performed using a GE Signa HDxt 3.0T scanner (GE Healthcare, Milwaukee, Wisc.) A T1-weighted image showed hyperintensity in the right putamen (Figure 1A). No significant signal abnormality was detected on T2-weighted, fluid attenuation and inversion recovery and gradient echo images except that diffusion-weighted images showed slightly elevated signal intensity. Serum levels of vitamin E, blood cell acanthocytosis, and electroencephalography were normal.

We initiated a twice-daily, premixed insulin preparation, and, after 1 week, normal glycemia was restored. The patient's movement disorder decreased in severity and completely disappeared 10 days after the first medical appointment. At his 2-month follow-up, despite symptomatic resolution, a repeat brain MRI showed the abnormalities that were seen on previous imaging. However, a 6-month re-scan with MRI showed a normal putamen without the previously seen hyperintense lesion (Figure 1B).

\section{Questions}

1. Why is it important to screen elderly patients with involuntary movements for hyperglycemia?

2. How can nonketotic hyperglycemia lead to $\mathrm{HCHB}$ ?

3. What radiological clues help to confirm the diagnosis of diabetic $\mathrm{HCHB}$ ?

4. Why is early recognition of this clinical entity is important?

\section{Commentary}

Nonketotic hyperglycemia is a rare cause of $\mathrm{HCHB}$, usually occurring in elderly, and especially Asian, women with type 2 diabetes (1). HCHB may be a complication in patients already known to have diabetes $(2,3)$, or it may be the first manifestation of the syndrome (4-6). In this case, HCHB was the presenting symptom of nonketotic diabetes.
The pathogenesis of this condition is thought to be related to hyperglycemia-induced perfusion changes in the contralateral striatum. It has been reported that hyperglycemia shifts cerebral metabolism to an anaerobic pathway and that striatal GABAergic neurons are particularly vulnerable to ischemia (7). The selective loss of striatal GABAergic neurons may be related to disinhibition of the thalamocortical pathway, resulting in the motor cortical hyperexcitability (4). Recently, it was suggested that the presence of acanthocytes in circulating peripheral blood might render people with diabetes prone to develop HCHB (8).

The diagnosis is made in the context of typical clinical and radiological features, including the presence of ballistic or choreiform movements in the setting of marked hyperglycemia and the absence of ketoacidosis. Neuroradiological findings of $\mathrm{HCHB}$ are particular and involve the putamen in all cases, the head of the caudate nucleus in most cases, and the globus pallidus in a minority of cases. Lesions are unilateral in the vast majority of cases, although bilateral involvement has been reported $(3,4)$. The characteristic finding on a T1-weighted MRI is high signal intensity in the contralateral putamen without signs of mass effect, edema, or volume loss. In contrast, the findings in T2-weighted images are variable, with signal characteristics ranging from hyper- and iso- to hypointensity. Gradientecho and gadolinium-enhanced T1-weighted images are reported to be normal.

In the literature, it has been postulated that striatal hyperintensity in T1-weighted MRI could be the result of partial ischemic injury related to hyperglycemia-induced hyperviscosity. Single photon emission computed tomography and positron emission tomography studies have suggested that the metabolic derangements associated with hyperglycemia and vascular insufficiency contribute to regional metabolic failure in patients with poorly controlled diabetes $(5,9)$. The resulting hypoxic-ischemic damage induces metabolic derangements to which astrocytes react, causing proliferation of gemistocytes (swollen reactive astrocytes). Magnetic resonance spectroscopy evidence of decreased n-acetylaspartate (NAA) and creatine $(\mathrm{Cr})$ peaks with a low NAA/Cr ratio (10) suggests neuronal dysfunction, loss, or damage.

Neuroimaging findings may return to normal or persist after clinical recovery, as occurred in this case (4). The mainstay of treatment is aggressive glycemic control with recovery from clinical symptoms after normalization of hyperglycemia. In refractory cases, additional treatment with haloperidol, risperidone, or tetrabenazine have been discussed as an option to improve the movement disorder (11). Recognition of this unique clinicoradiological manifestation is important because correction of the underlying hyperglycemia will lead to rapid improvement. Screening all patients who present with involuntary movements for hyperglycemia, even those without a history of diabetes, is important.

\section{Clinical Pearls}

- HCHB associated with nonketotic hyperglycemia is an uncommon manifestation of diabetes, usually occurring in elderly, and especially Asian, women.

- All patients with this clinical presentation should be screened for diabetes because $\mathrm{HCHB}$ may be the first manifestation of the syndrome.

- It is typically accompanied by characteristic brain imaging findings contralateral to the side of neurological abnormalities.

- Prompt diagnosis and treatment of hyperglycemia leads to complete resolution of the symptoms and signs of HCHB.

- HCHB syndrome should be regularly included in the differential diagnosis of acute movement dis- 
orders, especially in the elderly population.

\section{Duality of Interest}

No potential conflicts of interest relevant to this article were reported.

\section{References}

1. Dewey RB Jr, Jankovic J. Hemiballismhemichorea: clinical and pharmacologic findings in 21 patients. Arch Neurol 1989;46:862-867

2. Crausman RS, Wen J, Al-Shalabi S. Choreoathetosis and diabetes. Diabetes Care 1997;20:1209-1210

3. Higa M, Kaneko Y, Inokuchi T. Two cases of hyperglycaemic chorea in diabetic patients. Diabet Med 2004;21:196-198
4. Shan DE, Ho DM, Chang C, Pan HC, Teng MM. Hemichorea-hemiballism: an explanation for MR signal changes. Am J Neuroradiol 1998;19:863-870

5. Lee EJ, Choi JY, Lee SH, Song SY, Lee YS. Hemichorea-hemiballism in primary diabetic patients: MR correlation. J Comput Tomogr 2002;26:905-911

6. Lin JJ, Lin GY, Shih C, Shen WC. Presentation of striatal hyperintensity on T1-weighted MRI in patients with hemiballism-hemichorea caused by nonketotic hyperglycemia: report of seven new cases and a review of literature. J Neurol 2001;248:750-755

7. Oh SH, Lee KY, Im JH, Lee MS. Chorea associated with non-ketotic hyperglycemia and hyperintensity basal ganglia lesion on T1-weighted brain MRI study: a meta-anal- ysis of 53 cases including four present cases. J Neurol Sci 2002;200:57-62

8. Pisani A, Diomedi M, Rum A, et al. Acanthocytosis as a predisposing factor for non-ketotic hyperglycaemia induced chorea-ballism. J Neurol Neurosurg Psychiatry 2005;76:1717-1719

9. Hsu JL, Wang HC, Hsu WC.

Hyperglycemia-induced unilateral basal ganglion lesions with and without hemichorea: a PET study. J Neurol 2004;251:1486-1490

10. Lai PH, Chen PC, Chang MH, et al. In vivo proton MR spectroscopy of chorea-ballismus in diabetes mellitus. Neuroradiology 2001; $43: 525-531$

11. Battisti C, Forte F, Rubenni E, et al. Two cases of hemichorea-hemiballism with nonketotic hyperglycaemia: a new point of view. Neurol Sci 2009;30:179-183 